\title{
FORUM
}

\section{EU-Erweiterungspolitik mit Augenmaß: eine deutsch-britische Perspektive}

\author{
Günter Gloser und Glenys Kinnock*
}

Am 1. Mai 2009 haben wir fünf Jahre Osterweiterung gefeiert und dies war wahrlich ein Grund zu feiern: Die Aufnahme von zehn neuen Mitgliedstaaten stellte die größte Erweiterung in der Geschichte der Europäischen Union dar. Ein historisches Ereignis. Wir stehen vor dem Ergebnis einer gewaltigen gesellschaftlichen, politischen und wirtschaftlichen Transformation. Die Europäische Union hat hierbei bewiesen, dass sie sich zum Motor dieser Transformation machen kann, wenn - und nur wenn - mutige Bürger/innen und entschlossene Politiker/innen bereit sind, diesen Weg zu gehen. Das, was wir heute in den neuen Mitgliedstaaten sehen, oft beschrieben und nach fünf Jahren ein Stück Normalität geworden, bleibt dennoch nicht weniger eindrucksvoll: der Umbau sozialistischer Plan- in Marktwirtschaften, der Aufbau demokratischer Institutionen, die Stärkung von Menschenrechten und Rechtsstaatlichkeit, sozialer Fortschritt. Und wenn auch die aktuelle Wirtschafts- und Finanzkrise bei den neuen Partnern einen Schatten auf die positive Entwicklung der letzten Jahre wirft. Die tatkräftige Unterstützung der Europäischen Union beweist, dass die Probleme in der Gemeinschaft leichter zu lösen sein werden.

Jetzt gilt es, diese Transformationskraft der Europäischen Union zu bewahren. Wir brauchen in der Zukunft eine Erweiterungspolitik mit Augenmaß. Der Beitritt zur Union ist kein Allheilmittel. Er setzt einen Prozess schwieriger und schmerzhafter Reformen voraus. Regelmäßig müssen liebgewonnene Besitzstände aufgegeben werden. Die Wirtschaft fürchtet den Konkurrenzdruck; Marktanteile werden neu verteilt. Befürchtungen vor dem Verlust von Arbeitsplätzen, aber auch steigender Kriminalität und illegaler Migration machen die Runde. Hier gilt es, die Fakten von den Mythen zu trennen. Von der Öffnung der Märkte in Mittel- und Osteuropa haben gerade exportorientierte Volkswirtschaften wie die Großbritanniens und Deutschlands besonders profitiert. Empirische Studien belegen die Wohlstandsgewinne durch den vergrößerten Binnenmarkt. Was als kritische Entwicklung wahrgenommen wird, ist oft Folge der Globalisierung und nicht die vermeintliche Konsequenz einer erweiterten Union. Im Gegenteil: Die Erweiterung eröffnet uns die Chance, den Globalisierungsprozess mitzugestalten. Wir müssen ,Europa kommunizieren“ wie es oft im EU-Jargon heißt - und gerade unsere neuen Partner können dies mit besonderer Glaubwürdigkeit.

Eine Erweiterungspolitik mit Augenmaß heißt sicherzustellen, dass die Bedingungen, die wir für den Beitritt neuer Mitglieder gesetzt haben, ohne Abstriche erfüllt werden. Wir wissen, dass diese Kriterien und die Anforderungen an die Beitrittsreife neuer Kandidaten anspruchsvoll sind. Sie müssen es sein, denn wir brauchen den Reformdruck. Defizite in Verwaltung und Justiz oder ,Löcher' bei der Bekämpfung von Organisierter Kriminalität und Korruption etwa sind nicht hinnehmbar. Diese Bereiche sind essenziell für die spätere Umsetzung des Gemeinschaftsacquis. Länder, die beitreten wollen, brauchen feste politische, wirtschaftliche und administrative Strukturen, um die mit der Mitgliedschaft einhergehen-

Günter Gloser, MdB, Staatsminister für Europa, Auswärtiges Amt, Berlin.

Baroness Kinnock of Holyhead, Mitglied des House of Lords, Staatsministerin (Europe), London. 
den Verpflichtungen umzusetzen. Es kann hierbei weder Abkürzungen noch Rabatte geben. Striktes Festhalten am Prinzip der Konditionalität schafft letztlich jene Glaubwürdigkeit und jenes Vertrauen, das wir beim Bürger dringend brauchen, um die europäische Integration erfolgreich voranzutreiben.

Eine Erweiterungspolitik mit Augenmaß heißt, dass wir zu den Verpflichtungen stehen, die wir gegenüber der Türkei, Kroatien und den übrigen Ländern des Westlichen Balkans ausgesprochen haben. Auch dies ist ein Gebot der Glaubwürdigkeit. Die europäische Perspektive des Westlichen Balkans ist seit dem Gipfel von Thessaloniki 2003 vielfach durch die Staats- und Regierungschefs bestätigt worden. Eine Perspektive, die selbstverständlich für den gesamten Westlichen Balkan gilt, einschließlich Kosovo. Europa braucht auf dem Balkan zu seiner eigenen Sicherheit dauerhaften Frieden und wirtschaftlich gesunde Verhältnisse. Die abgeschlossenen Stabilisierungs- und Assoziierungsabkommen bilden hierfür eine ausgezeichnete Grundlage. Sie müssen nach Geist und Buchstabe umgesetzt werden. Nur wenn Erfahrungen in der Implementierung und Zusammenarbeit über einen längeren Zeitraum gesammelt werden können, sind belastbare Rückschlüsse über die Beitrittsreife eines Landes möglich. Im Reformprozess bleibt letztlich jedes Land seines eigenen Glückes Schmied.

Die Türkei ist ein Verbündeter und Partner, der seit den 60er-Jahren über den Assoziationsvertrag und später die Zollunion (1995) mit der Europäischen Union aufs Engste verbunden ist. Aber die Handelsinteressen greifen zu kurz. Das Potenzial dieses Landes an der Nahtstelle zu Asien sollten wir nicht unterschätzen. Ankara trägt erhebliche politische Verantwortung in einer krisengeschüttelten Region. Darüber hinaus beweist das Land täglich neu, wie eine funktionierende rechtsstaatliche Demokratie mit dem Islam, zu dem sich das türkische Volk in seiner überwiegenden Mehrheit bekennt, in Einklang gebracht werden kann. Die Türkei hat eine zentrale Rolle als Transitland zur Sicherung des europäischen Energiebedarfs. Es war daher richtig, dass die Europäische Union im Jahr 2005 Beitrittsverhandlungen aufgenommen hat und wir wollen diese kontinuierlich fortsetzen - das Ziel des Beitritts vor Augen. Was wir hierbei brauchen, ist eine aktive türkische Regierung, die - unter Einbeziehung des Parlaments und der Zivilgesellschaft - gleichermaßen beherzt auf dem Reformweg voranschreitet. Wenn wir dieses Ziel infrage stellen, ist den Verhandlungen die Basis entzogen.

Eine Erweiterungspolitik mit Augenmaß heißt daher auch, dass wir als Union in der Lage sind, begonnene Beitrittsverhandlungen zu Ende führen zu können. Es zeigt sich aber, dass die Beitrittsverhandlungen zunehmend unter nationalen Vorbehalten leiden, die uns daran hindern voranzuschreiten. Es ist uns ein starkes Anliegen, dass bilaterale Probleme nicht in die Beitrittsverhandlungen hineingetragen werden.

Eine Erweiterungspolitik mit Augenmaß heißt viertens, dass wir innerhalb der Europäischen Union die notwendigen Voraussetzungen schaffen, damit wir im größeren Kreis gleichberechtigter Mitgliedstaaten auch weiterhin effektiv und erfolgreich zusammenarbeiten können. Wir brauchen mehr innere Kohäsion und die vielzitierte, eine Stimme'. Nur dies gibt uns Gewicht bei der Lösung der großen internationalen Aufgaben; Klimaschutz steht hier Pars pro Toto. Hierfür brauchen wir den Vertrag von Lissabon. Er sichert das notwendige ,Mehr' an Effizienz und Kohärenz, aber auch Demokratie und Bürgernähe. Dies liegt im Interesse aller: der aktuellen wie auch zukünftiger Mitgliedstaaten.

Großbritannien und Deutschland werden auch weiterhin für eine Erweiterungspolitik mit Augenmaß eintreten. Es geht hierbei um nicht weniger als die Wahrung von Sicherheit, Wohlstand und Stabilität für die Zukunft Europas. 